\title{
Characteristics of 151 Brazilian Sporothrix schenckii Isolates from 5 Different Geographic Regions of Brazil: A Forgotten and Re-Emergent Pathogen
}

\author{
Geisa Ferreira Fernandes ${ }^{1}$, Priscila Oliveira dos $\operatorname{Santos}^{1}$, Cristiane Candida do Amaral ${ }^{1}$, \\ Alexandre Augusto Sasaki ${ }^{1}$, Patricio Godoy-Martinez ${ }^{1,2}$ and Zoilo Pires de Camargo ${ }^{*},{ }^{1}$ \\ ${ }^{1}$ Federal University of São Paulo (UNIFESP), São Paulo, SP, Brazil \\ ${ }^{2}$ Discipline of Infectious and Parasitic Diseases, IMC Universidad Austral de Chile, Valdivia, Chile
}

\begin{abstract}
We used RAPD (random amplification of polymorphic DNA) to analyze the phenotypic and genotypic characteristics of Sporothrix schenckii isolated from five geographic regions of Brazil, from clinical, animal, and environmental sources. Our results yielded a significant difference $(\mathrm{P}<0.01)$ in the mean conidial area of $S$. schenckii animal isolates $(2.96 \pm 1.07)$ compared with those of clinical isolates (fixed form, $2.33 \pm 0.53$; lymphocutaneous form, $2.37 \pm 0.43)$. There was no association among $S$. schenckii clinical isolates and geographic region. Isolates from the Northeast region exhibited the lowest thermotolerance (\% growth inhibition) at $35^{\circ} \mathrm{C}(\bar{x}=49.23 \% \pm 17.25)$ and at $37^{\circ} \mathrm{C}$ $(70.43 \% \pm 10.93 \%)$. Northern isolates exhibited the highest thermotolerance at $35^{\circ} \mathrm{C}(12.82 \% \pm 5.73 \%)$ and at $37^{\circ} \mathrm{C}$ $(23.81 \% \pm 8.27 \%)$. RAPD with a 10-mer primer OPD-18 generated 67 PCR fingerprint patterns. The 151 S. schenckii isolates fell into seven major clusters with such great genetic diversity that an association of isolates with clinical forms or geographic areas could not be determined, even with investigations focused on more restricted geographic areas. The main physiological characteristics of Brazilian S. schenckii isolates were also characterized, including osmophilia, halophilia, $\mathrm{pH}$ tolerance, urease activity, casein hydrolysis, and gelatinase, proteinase, and DNAase production.
\end{abstract}

Keywords: Sporothrix schenckii, sporotrichosis, molecular biology, physiology, epidemiology.

\section{INTRODUCTION}

Sporothrix schenckii is a dimorphic fungus found worldwide as a pathogenic agent of sporotrichosis, a chronic infection of the skin and subcutaneous tissues, characterized by the development of lymphatic nodules in humans and other animals [1-3]. The disease can disseminate, affecting any organ of the human body [1-3]. Generally, infection results from inoculation of the fungus during trauma and is related to occupational activities [1,3-5]. In recent years, the incidence of the disease has gradually increased in humans, mostly among veterinarians, nurses, and cat owners, who are usually infected through cat scratches or bites or even by direct contact with exudates of feline lesions [6]. The disease affects all age groups [3, 7], and the number of cases involving men and women varies from region to region $[3,6$, $8,9]$, possibly depending on the type of fungal exposure [10]. The fungus has been isolated from soil, various plant species, and sphagnum moss $[1,11,12]$, and when it is introduced into the host, the mycelial form changes to the yeast cells, the infective form $[1,3,4]$. For clinical diagnosis of sporotrichosis, it is necessary to isolate $S$. schenckii in culture from clinical material. In general, it is identified

*Address correspondence to this author at the Federal University of São Paulo (UNIFESP), Department of Microbiology, Immunology and Parasitology, Discipline of Cellular Biology. 04023-062 Rua Botucatu 862/8º, São Paulo, SP, Brazil; Tel: 55115576 4523;Fax; 55115571 5877; E-mail: zpcamargo@unifesp.br based on the morphological characteristics of its colonies, conidia, and other structures in vitro $[1,3,4]$.

In South America, the countries of Brazil, Uruguay, Peru, and Colombia constitute areas of $S$. schenckii endemicity [5, 10]. In Brazil, isolated cases, small outbreaks, and case series have been sporadically reported [13- 15]. In general, very little has been published in recent decades about clinical cases of sporotrichosis in Brazil; however, since 1998, the research group at the Evandro Chagas Clinical Research Institute, Fiocruz, in Rio de Janeiro, has diagnosed 1503 cats, 64 dogs, and 759 people with sporotrichosis confirmed by isolation of $S$. schenckii in culture [6,16-18].

Because cases of sporotrichosis do not have to be reported, the real incidence of the disease in Brazil is unknown; thus, there is little information about its true prevalence. However, over the last 3 years, we collected 151 $S$. schenckii isolates from five different regions of Brazil, a continental country (with an area of $8,514,204.8 \mathrm{~km}^{2}$ ): the North, Northeast, Center West, Southeast, and South. These strains were isolated in 14 of 26 Brazilian states, indicating indirectly that the actual prevalence of sporotrichosis is higher than we had supposed. Parts of the southeastern and southern regions are influenced by polar air masses, whereas the northern and northeastern regions are influenced by hot air masses so that the annual temperature is continuously high. Despite the clinical importance of $S$. schenckii, little is known about its basic biology and population structure among Brazilian isolates. 
Recently, work has shown that traditional species of $S$. schenckii exhibit a high genetic variability, and new species have been described within the "Sporothrix schenckii aggregate" [19-21]. Among these new species, S. brasiliensis has been found only in Brazil $[19,20]$. These new species were based on the sequence analysis of three protein coding loci (chitin synthase, $\beta$-tubulin, and calmodulin). The Spanish group of Dr. Josep Guarro proposed identifying the Brazilian S. schenckii strains as $S$. brasiliensis $[19,20]$; in this paper, however, we refer to this fungus by its traditional name of $S$. schenckii because we did not perform molecular phylogenetic analysis of our isolates in the context of DNA sequence data from the three different loci cited above.

Recently, Mesa-Arango et al. [22] studied 44 clinical and environmental isolates of $S$. schenckii from Mexico, Guatemala, and Colombia. In this well-elaborated work, the authors examined the phenotypic characterization - conidial measurements, thermotolerance, aspects of virulence and genotyping characteristics - by means of random amplification of polymorphic DNA (RAPD) analysis [22].

In the current study, our aim was to determine the phenotypic relatedness [conidial size and thermotolerance by percent growth inhibition (GI) at $35^{\circ} \mathrm{C}$ and $37^{\circ} \mathrm{C}$ ] among 151 Brazilian S. schenckii isolates from five different geographic regions of this continental country with distinct climates. Further, we investigated the molecular epidemiology using the RAPD technique. Our study was based on Mesa-Arango et al. [22] models; however, only Brazilian S. schenckii isolates were analyzed, and the data reported here constitute the first study of this type in Brazil. Because $S$. schenckii has been poorly studied for its physiological characteristics, we selected a representative set of 19 Brazilian isolates of $S$. schenckii from different geographic regions of Brazil and analyzed them according to the methods described by Ghosh et al. [23] as the basis for that study.

\section{MATERIALS AND METHODOLOGY}

\section{Fungal Isolates}

This study included 151 Brazilian isolates of S. schenckii (139 from clinical sources; 10 from felines, one from a dog, and one from an environmental source) (Table 1). By region, 3 isolates were from the North, 17 from the Northeast, 8 from the Center West, 84 from the Southeast, and 39 from the South. All clinical isolates were from patients with the fixed (76 cases), lymphocutaneous (60 cases), extracutaneous ( 1 case), or disseminated form ( 2 cases) of sporotrichosis. Isolates were stored on potato dextrose agar (Difco Laboratories, Detroit, $\mathrm{MI}$ ) at $4^{\circ} \mathrm{C}$ to $7^{\circ} \mathrm{C}$ and deposited in the fungal collection of the Laboratory of Fungal Diseases of the Discipline of Cellular Biology, Federal University of São Paulo.

\section{Isolate Identification}

The micro- and macromorphology of colonies were identified according to conventional methods. For the study of conidia formation and conidiogenous cells, slides were prepared using classical slide culture methods. Colony morphology was compared based on descriptions by Rippon [3].

\section{Phenotypic Characterization}

\section{Conidial Measurements}

The areas of the conidia were measured using the program UTHSCSA Image Tool 3.0. All measurements were estimated on the basis of the results obtained with at least 100 conidia/isolate from 15-day-old slide microcultures at room temperature.

\section{Thermotolerance}

Thermotolerance assays were performed as followed: the percent growth inhibition (GI) was calculated at 35 and $37^{\circ} \mathrm{C}$ by the following formula: [(colony diameter at $28^{\circ} \mathrm{C}$ - colony diameter at 35 or $37^{\circ} \mathrm{C}$ )/colony diameter at $28^{\circ} \mathrm{C}$ ] x 100 as proposed by Mesa-Arango et al. [22].

\section{Molecular Fingerprint Method: RAPD}

Isolation of S. schenckii DNA and RAPD assays were performed according to protocols previously described by Liu et al. [24]. The program for PCR reactions was that proposed by Liu et al. [24], and the primers used were: OPA11 (5'-ACCCGACCTG-3'), OPD18 (5'GAGAGCCAAC-3'), and OPB07 (5'-GGTGACGCAG-3') (Operon Technologies Ltda.) proposed by Liu et al. [24]; OPBG01 (5'-GTGGCTCTCC-3'), OPBG14 (5'GACCAGCCCA-3'), and OPBG19 (5'-GGTCTCGCTC-3') proposed by Mesa-Arango et al. [22]. The similarity was calculated by using the Dice coefficient, and the similarity matrix was the basis for the construction of a dendrogram using the unweighted pair-group method with arithmetic averages (UPGMA).

\section{Physiological Characteristics of $\boldsymbol{S}$. schenckii Isolates}

A representative set of 19 S. schenckii isolates-four each from the Northeast, Southeast, South, and Center West regions and three isolates from the North region-was chosen for physiological tests; 17 were of human origin, one of animal origin (cat), and one of environmental origin.

The laboratory procedures for assessing the physiological characteristics of osmophilia, halophilia, $\mathrm{pH}$ tolerance, urease activity, casein hydrolysis, and gelatinase production were performed according to Ghosh et al. [23] for $S$. schenckii, as briefly described below. For DNAase and proteinase activities, we followed the protocols proposed by Price et al. [25].

For osmophilia, we tested the isolates inoculated on media containing $16.6 \%, 20 \%, 23 \%$, or $28.5 \%$ glycerol; for halophilia, the fungus was inoculated on media containing increasing concentrations of sodium chloride $(6 \%, 8 \%, 9 \%$, $10 \%$, or $12 \%$ ); and for $\mathrm{pH}$ tolerance, we tested fungal growth at $\mathrm{pH} 2.2,4.0,12.0,12.5$, and 13.0.

\section{RESULTS}

\section{Phenotypic Characterization}

All isolates studied presented typical micro- and macrocharacteristics of $S$. schenckii similar to those described by Rippon [3].

Table 1 shows the phenotypic characteristics studied for all isolates, specifically the conidium size, measured in area 
Table 1. The S. schenckii Isolates Studied and their Phenotypic Characteristics

\begin{tabular}{|c|c|c|c|c|c|}
\hline \multirow{2}{*}{\multicolumn{2}{|c|}{ Isolate no. }} & \multirow{2}{*}{ Source $^{c}$} & \multirow{2}{*}{ Conidial Size $\left(\text { Area } \mu \mathrm{m}^{2}\right)^{\mathrm{a}}$} & \multicolumn{2}{|c|}{ Thermotolerance $\left(\%\right.$ GI) ${ }^{b}$ at: } \\
\hline & & & & $35^{\circ} \mathrm{C}$ & $37^{\circ} \mathrm{C}$ \\
\hline \multicolumn{6}{|l|}{ Southest region } \\
\hline & Ss-1 & Cat, São Paulo & 2.09 & 24.48 & 29.20 \\
\hline & Ss-52 & F, São Paulo & 1.89 & 31.84 & 54.46 \\
\hline & Ss-58 & F, São Paulo & 2.10 & 41.14 & 66.67 \\
\hline & Ss-59 & L, São Paulo & 2.65 & 51.16 & 53.49 \\
\hline & Ss-60 & L, São Paulo & 2.49 & 29.54 & 69.32 \\
\hline & Ss-61 & Soil, São Paulo & 2.60 & 34.79 & 54.12 \\
\hline & Ss-101 & L, São Paulo & 2.28 & 41.25 & 62.50 \\
\hline & Ss-102 & L, São Paulo & 2.17 & 60.40 & 72.28 \\
\hline & Ss-103 & L, São Paulo & 2.46 & 46.07 & 65.17 \\
\hline & Ss-111 & F, São Paulo & 2.08 & 34.72 & 84.72 \\
\hline & Ss-112 & F, São Paulo & 2.49 & 39.39 & 79.80 \\
\hline & Ss-113 & F, São Paulo & 2.66 & 30.59 & 47.06 \\
\hline & Ss-114 & F, São Paulo & 2.03 & 30.49 & 51.22 \\
\hline & Ss-115 & F, São Paulo & 2.67 & 45.74 & 71.28 \\
\hline & Ss-116 & F, São Paulo & 2.15 & 61.54 & 66.67 \\
\hline & Ss-117 & F, São Paulo & 2.64 & 61.54 & 64.84 \\
\hline & Ss-118 & F, São Paulo & 2.48 & 19.75 & 35.80 \\
\hline & Ss-119 & EC, São Paulo & 2.19 & 67.50 & 71.25 \\
\hline & Ss-120 & F, São Paulo & 2.38 & 65.56 & 75.56 \\
\hline & Ss-121 & F, São Paulo & 2.20 & 65.12 & 69.77 \\
\hline & Ss-122 & F, São Paulo & 2.45 & 73.33 & 85.33 \\
\hline & Ss-123 & F, São Paulo & 3.01 & 77.00 & 82.00 \\
\hline & Ss-124 & F, São Paulo & 2.22 & 74.49 & 74.49 \\
\hline & Ss-125 & F, São Paulo & 2.01 & 61.76 & 63.24 \\
\hline & Ss-126 & F, São Paulo & 2.58 & 71.59 & 77.27 \\
\hline & Ss-127 & F, São Paulo & 2.80 & 66.67 & 71.11 \\
\hline & Ss-128 & F, São Paulo & 3.20 & 50.00 & 65.79 \\
\hline & Ss-129 & L, São Paulo & 2.26 & 56.25 & 75.00 \\
\hline & Ss-148 & L, São Paulo & 2.70 & 46.88 & 65.63 \\
\hline & Ss-65 & F, Rio de Janeiro & 2.10 & 15.47 & 65.33 \\
\hline & Ss-66 & F, Rio de Janeiro & 2.45 & 36.10 & 56.87 \\
\hline & Ss-67 & F, Rio de Janeiro & 2.28 & 21.88 & 29.69 \\
\hline & Ss-68 & F, Rio de Janeiro & 2.20 & 48.11 & 52.83 \\
\hline & Ss-69 & F, Rio de Janeiro & 2.10 & 16.27 & 55.62 \\
\hline & Ss-70 & F, Rio de Janeiro & 2.05 & 24.89 & 45.70 \\
\hline & Ss-71 & L, Rio de Janeiro & 2.28 & 18.12 & 32.61 \\
\hline & Ss-72 & F, Rio de Janeiro & 2.41 & 18.94 & 42.26 \\
\hline & Ss-73 & F, Rio de Janeiro & 2.29 & 19.08 & 35.20 \\
\hline & Ss-74 & F, Rio de Janeiro & 2.39 & 36.23 & 57.39 \\
\hline & Ss-75 & F, Rio de Janeiro & 2.44 & 38.78 & 59.18 \\
\hline & Ss-76 & F, Rio de Janeiro & 2.12 & 36.33 & 66.21 \\
\hline
\end{tabular}


(Table 1) contd.....

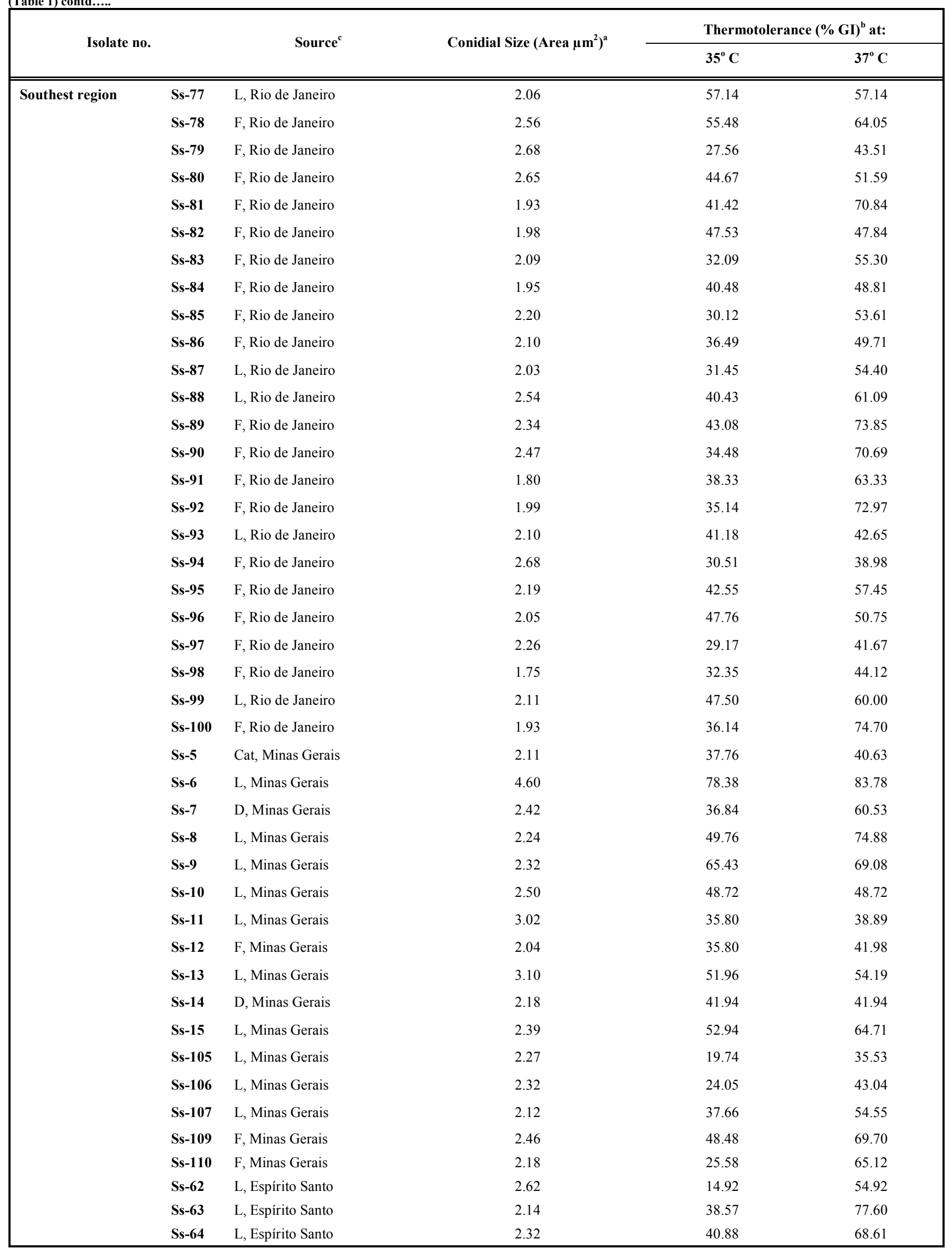


(Table 1) contd......

\begin{tabular}{|c|c|c|c|c|c|}
\hline \multirow{2}{*}{ Isolate no. } & & \multirow{2}{*}{ Source $^{c}$} & \multirow{2}{*}{ Conidial Size $\left(\text { Area } \mu \mathrm{m}^{2}\right)^{\mathrm{a}}$} & \multicolumn{2}{|c|}{ Thermotolerance $(\% \text { GI })^{\mathrm{b}}$ at: } \\
\hline & & & & $35^{\circ} \mathrm{C}$ & $37^{\circ} \mathrm{C}$ \\
\hline \multicolumn{6}{|c|}{ South region } \\
\hline & Ss-2 & F, Rio Grande do Sul & 2.96 & 62.50 & 77.00 \\
\hline & Ss-3 & F, Rio Grande do Sul & 2.46 & 40.91 & 53.41 \\
\hline & Ss-4 & F, Rio Grande do Sul & 2.33 & 26.67 & 39.49 \\
\hline & Ss-53 & Cat, Rio Grande do Sul & 2.14 & 22.15 & 36.08 \\
\hline & Ss-54 & Cat, Rio Grande do Sul & 5.29 & 29.29 & 60.36 \\
\hline & Ss-55 & L, Rio Grande do Sul & 2.19 & 19.41 & 55.70 \\
\hline & Ss-56 & L, Rio Grande do Sul & 2.23 & 23.53 & 44.12 \\
\hline & Ss-57 & L, Rio Grande do Sul & 2.60 & 15.04 & 49.19 \\
\hline & Ss-144 & L, Rio Grande do Sul & 2.30 & 46.67 & 64.44 \\
\hline & Ss-145 & L, Rio Grande do Sul & 2.82 & 42.55 & 53.19 \\
\hline & Ss-149 & L, Rio Grande do Sul & 2.30 & 42.86 & 42.86 \\
\hline & Ss-150 & L, Rio Grande do Sul & 3.09 & 34.43 & 34.43 \\
\hline & Ss-151 & Dog, Rio Grande do Sul & 2.64 & 13.04 & 34.78 \\
\hline & Ss-152 & Cat, Rio Grande do Sul & 3.36 & 38.60 & 47.37 \\
\hline & Ss-153 & Cat, Rio Grande do Sul & 2.10 & 30.00 & 36.00 \\
\hline & Ss-154 & Cat, Rio Grande do Sul & 3.11 & 27.27 & 41.82 \\
\hline & Ss-155 & Cat, Rio Grande do Sul & 3.19 & 38.60 & 43.86 \\
\hline & Ss-156 & Cat, Rio Grande do Sul & 2.10 & 31.03 & 39.66 \\
\hline & Ss-157 & Cat, Rio Grande do Sul & 4.40 & 30.91 & 45.45 \\
\hline & Ss-17 & F, Paraná & 2.20 & 47.50 & 57.50 \\
\hline & Ss-19 & L, Paraná & 2.31 & 80.00 & 80.00 \\
\hline & Ss-20 & F, Paraná & 2.06 & 51.61 & 58.06 \\
\hline & Ss-21 & L, Paraná & 2.29 & 29.85 & 40.30 \\
\hline & Ss-22 & F, Paraná & 2.40 & 39.56 & 55.06 \\
\hline & Ss-24 & F, Paraná & 2.08 & 76.02 & 84.96 \\
\hline & Ss-25 & F, Paraná & 2.07 & 38.10 & 64.29 \\
\hline & Ss-26 & F, Paraná & 2.07 & 50.48 & 61.34 \\
\hline & Ss-27 & L, Paraná & 1.82 & 41.90 & 67.25 \\
\hline & Ss-28 & F, Paraná & 3.39 & 64.95 & 73.63 \\
\hline & Ss-30 & F, Paraná & 3.32 & 33.33 & 46.67 \\
\hline & Ss-31 & F, Paraná & 2.16 & 18.07 & 37.35 \\
\hline & Ss-32 & F, Paraná & 1.79 & 51.22 & 70.73 \\
\hline & Ss-33 & L, Paraná & 1.77 & 50.00 & 55.88 \\
\hline & Ss-34 & F, Paraná & 2.10 & 50.00 & 62.50 \\
\hline & Ss-35 & F, Paraná & 2.69 & 53.49 & 65.12 \\
\hline & Ss-36 & F, Paraná & 5.65 & 21.62 & 39.19 \\
\hline & Ss-37 & F, Paraná & 2.03 & 36.59 & 43.90 \\
\hline & Ss-38 & F, Paraná & 2.35 & 12.58 & 51.57 \\
\hline & Ss-39 & F, Paraná & 1.59 & 55.56 & 68.89 \\
\hline \multicolumn{6}{|l|}{ Center West region } \\
\hline & Ss-45 & L, Goiás & 2.10 & 16.72 & 52.90 \\
\hline & Ss-46 & L, Goiás & 2.02 & 30.49 & 39.02 \\
\hline & Ss-47 & L, Goiás & 1.62 & 18.43 & 76.79 \\
\hline & Ss-48 & L, Goiás & 2.58 & 30.00 & 41.43 \\
\hline & Ss-49 & L, Goiás & 1.88 & 65.71 & 65.71 \\
\hline & Ss-50 & L, Goiás & 2.25 & 41.18 & 61.76 \\
\hline & Ss-104 & L, Mato Grosso & 2.05 & 37.84 & 59.46 \\
\hline & Ss-141 & L, Distrito Federal & 2.54 & 42.55 & 72.34 \\
\hline
\end{tabular}


(Table 1) contd.....

\begin{tabular}{|c|c|c|c|c|c|}
\hline \multirow{2}{*}{ Isolate no. } & & \multirow{2}{*}{ Source $^{\mathrm{c}}$} & \multirow{2}{*}{ Conidial Size $\left(\text { Area } \mu \mathrm{m}^{2}\right)^{\mathrm{a}}$} & \multicolumn{2}{|c|}{ Thermotolerance $\left(\%\right.$ GI) ${ }^{\mathrm{b}}$ at: } \\
\hline & & & & $35^{\circ} \mathrm{C}$ & $37^{\circ} \mathrm{C}$ \\
\hline \multirow[t]{3}{*}{ North region } & Ss-51 & L, Pará & 2.34 & 6.93 & 33.33 \\
\hline & Ss-143 & L, Pará & 2.60 & 13.16 & 19.74 \\
\hline & Ss-158 & L, Amazonas & 2.00 & 18.37 & 18.37 \\
\hline \multicolumn{6}{|l|}{ Northeast region } \\
\hline & Ss-16 & L, Piauí & 2.12 & 34.18 & 43.04 \\
\hline & Ss-40 & F, Ceará & 1.65 & 12.84 & 61.20 \\
\hline & Ss-41 & F, Ceará & 2.17 & 54.29 & 62.86 \\
\hline & Ss-42 & F, Ceará & 1.69 & 67.49 & 68.85 \\
\hline & Ss-43 & F, Ceará & 2.08 & 11.19 & 71.84 \\
\hline & Ss-44 & L, Ceará & 1.71 & 58.82 & 64.71 \\
\hline & Ss-130 & L, Pernambuco & 2.50 & 33.33 & 70.67 \\
\hline & Ss-131 & L, Pernambuco & 2.52 & 61.25 & 77.50 \\
\hline & Ss-132 & L, Pernambuco & 2.02 & 61.82 & 87.27 \\
\hline & Ss-133 & L, Pernambuco & 2.20 & 63.81 & 89.52 \\
\hline & Ss-134 & L, Pernambuco & 2.13 & 67.21 & 78.69 \\
\hline & Ss-135 & L, Pernambuco & 2.66 & 60.53 & 72.37 \\
\hline & Ss-136 & L, Pernambuco & 2.70 & 58.14 & 77.91 \\
\hline & Ss-137 & L, Pernambuco & 2.78 & 47.06 & 65.88 \\
\hline & Ss-138 & L, Paraíba & 2.58 & 46.15 & 61.54 \\
\hline & Ss-139 & L, Paraíba & 2.31 & 48.78 & 76.83 \\
\hline & Ss-140 & L, Paraíba & 2.30 & 50.00 & 66.67 \\
\hline
\end{tabular}

${ }^{a}$ A minimum of 100 conidia from each isolate from 15-day-old PDA slide microcultures grown for 15 days at room temperature were measured.

${ }^{\mathrm{b}}$ Percent growth inhibition was calculated in triplicate for samples from three different assays using the formula provided in the Materials and methodology section.

${ }^{\mathrm{c}} \mathrm{F}$, fixed form of sporotrichosis; L, lymphocutaneous form of sporotrichosis; EC, extracutaneous form of sporotrichosis; D, disseminated form of sporotrichosis.

$\left(\mu \mathrm{m}^{2}\right)$, and thermotolerance at $35^{\circ} \mathrm{C}$ and $37^{\circ} \mathrm{C}(\% \mathrm{GI})$ for all isolates. The conidial area of isolates varied from $1.59 \mu \mathrm{m}^{2}$ to $5.65 \mu \mathrm{m}^{2}$, while this area in cat and dog isolates was statistically different $(\mathrm{p} \leq 0.05)$ in size $\left(\mu \mathrm{m}^{2}\right)$ from human isolates (animal, $2.96 \mu \mathrm{m}^{2} \pm 1.07 \mu \mathrm{m}^{2}$ versus fixed form, $2.33 \mu \mathrm{m}^{2} \pm 0.53 \mu \mathrm{m}^{2}$ and lymphocutaneous form, $2.37 \mu \mathrm{m}^{2} \pm$ $0.43 \mu \mathrm{m}^{2}$ ). Using geographic region of origin as the parameter, no statistical differences were observed in relation to conidial area. Table 2 shows the mean $S$. schenckii conidial areas from each Brazilian region and \%GI at both $35^{\circ} \mathrm{C}$ and $37^{\circ} \mathrm{C}$.

The isolates had different thermotolerances at the two temperatures. At $35^{\circ} \mathrm{C}$, the $\% \mathrm{GI}$ values for the isolates were as follows: North, $\bar{x}=12.82 \% \pm 5.73 \%$; Northeast, $\bar{x}=$ $49.23 \% \pm 17.25 \%$; Center West, $\bar{x}=35.37 \% \pm 15.58 \%$; Southeast, $\bar{x}=41.89 \% \pm 15.34 \%$; and South, $\bar{x}=38.92 \% \pm$ $16.18 \%$. The isolates from the northern region were statistically different from the southern, southeastern, and northeastern regions. S. schenckii animal isolates were statistically different from those isolated from human lymphocutaneous forms: $(\bar{x}=29.38 \% \pm 7.70 \%)$ versus $(\bar{x}=$ $42.63 \% \pm 16.14 \%)$.

At $37^{\circ} \mathrm{C}$, the isolates exhibited the following $\% \mathrm{GI}$ values: North, $\bar{x}=23.81 \% \pm 8.27 \%$; Northeast, $\bar{x}=70.43 \% \pm$
$10.93 \%$; Center West, $\bar{x}=58.68 \% \pm 13.58 \%$; Southeast, $\bar{x}=58.83 \% \pm 13.81 \%$; and South, $\bar{x}=53.42 \% \pm 13.60 \%$. At $37^{\circ} \mathrm{C}$, the isolates from the North were statistically different from isolates from other regions, and isolates from the Northeast were statistically different from those of the South and Southeast.

On the other hand, at $37^{\circ} \mathrm{C}$, animal isolates $(\bar{x}=41.38 \%$ $\pm 8.19 \%$ ) differed statistically from human isolates [lymphocutaneous $(\bar{x}=59.45 \% \pm 15.90 \%)$ and fixed form $(\bar{x}=59.38 \% \pm 13.59 \%)]$.

\section{RAPD Genotypic Characterization}

The six primers tested yielded clearly scorable, repeatable, polymorphic banding patterns among the isolates. The patterns produced by RAPD analysis/PCR with primer OPD18 were the best that we obtained, consisting of 1 to 10 amplification bands ranging in size from 0.18 to $1.8 \mathrm{~kb}$. The similarities between isolate pairs were highly variable and ranged from $16.66 \%$ to $83.33 \%$. The mean genetic similarity was $49.99 \%$. Sixty-seven PCR fingerprinting patterns were generated, indicating a high discrimination among the $151 \mathrm{~S}$. schenckii isolated from different geographic regions. Genetic relationships obtained with the UPGMA method are represented as a dendrogram (Fig. 1), in which seven clusters can be distinguished. Cluster I was subdivided into Ia (5 
Table 2. Mean Conidial Area and Percent GI for Isolates from Different Regions of Brazil

\begin{tabular}{|c|c|c|c|}
\hline \multirow{2}{*}{ Isolates From } & \multirow{2}{*}{ Conidial Size (Area $\left.\mu \mathrm{m}^{2}\right)$} & \multicolumn{2}{|c|}{$\%$ GI at: } \\
\hline & & $35^{\circ} \mathrm{C}$ & $37^{\circ} \mathrm{C}$ \\
\hline Southeast & $2.34 \pm 0.38$ & $41.89 \pm 15.34$ & $58.83 \pm 13.81$ \\
\hline South & $2.61 \pm 0.88$ & $38.92 \pm 16.18$ & $53.42 \pm 13.60$ \\
\hline Center West & $2.13 \pm 0.32$ & $35.37 \pm 15.58$ & $58.68 \pm 13.58$ \\
\hline North & $2.31 \pm 0.30$ & $12.82 \pm 5.73$ & $23.81 \pm 8.27$ \\
\hline Northeast & $2.24 \pm 0.35$ & $49.23 \pm 17.25$ & $70.43 \pm 10.93$ \\
\hline \multirow{2}{*}{ Human/Animal } & \multirow{2}{*}{ Conidial Size (Area $\mu \mathbf{m}^{2}$ ) } & \multicolumn{2}{|c|}{$\%$ GI at: } \\
\hline & & $35^{\circ} \mathrm{C}$ & $37^{\circ} \mathrm{C}$ \\
\hline Fixed form & $2.33 \pm 0.53$ & $41.22 \pm 16.99$ & $59.38 \pm 13.59$ \\
\hline Lymphocutaneous & $2.37 \pm 0.43$ & $42.63 \pm 16.14$ & $59.45 \pm 15.90$ \\
\hline \multicolumn{4}{|l|}{ Form } \\
\hline Cats and dog & $2.96 \pm 1.07$ & $29.38 \pm 7.70$ & $41.38 \pm 8.19$ \\
\hline
\end{tabular}

GI, growth inhibition.

isolates) and $\mathrm{Ib}$ (142 isolates). Cluster $\mathrm{Ib}$ exhibited a clear separation into two subgroups: subgroup $\mathrm{Ib} .1$ accounted for 28 isolates presenting $52 \%$ relatedness, and subgroup $\mathrm{Ib} .2$ was composed of 114 isolates with $60 \%$ relatedness. Cluster $\mathrm{Ib} .2$ was also subdivided into Ib.2.1 with 20 isolates with $65 \%$ relatedness, and $\mathrm{Ib} .2 .2$ consisted of 94 isolates with $67 \%$ relatedness. Cluster Ib.2.2 was then subdivided into Ib.2.2.1 (77 isolates; 69\% relatedness) and Ib.2.2.2 (17 isolates; $74 \%$ relatedness). Cluster $\mathrm{Ib} .2 .2 .1$ was again subdivided into $\mathrm{Ib} \cdot 2.2 .1 .1$ (50 isolates; $74 \%$ relatedness) and Ib.2.2.1.2 (27 isolates; 76\% relatedness).

Because of the great diversity among all $S$. schenckii isolates from the different regions of Brazil, we decided to analyze those obtained in a smaller region (Sao Paulo State; area $=248,209.4 \mathrm{~km}^{2}$ ) and in a specific city (Rio de Janeiro; area $=1,255.3 \mathrm{~km}^{2}$ ). The fingerprint patterns obtained with OPD18 for each dataset are represented as a dendrogram and shown in Figs. $(\mathbf{2}, \mathbf{3})$, respectively. The fingerprint pattern obtained from $S$. schenckii isolates from Sao Paulo State (28 isolates) indicated three clusters. Cluster I was subdivided into Ia, composed of 18 isolates presenting $43 \%$ similarity, and $\mathrm{Ib}$ was composed of 8 isolates displaying $47 \%$ similarity; cluster II consisted of 2 isolates (Fig. 2). The dendrogram obtained with $36 \mathrm{~S}$. schenckii isolates from Rio de Janeiro showed three main clusters: cluster I consisted of 5 isolates with $39 \%$ relatedness; cluster II was subdivided into IIa consisting of 23 isolates presenting $51 \%$ similarity and IIb, comprising 8 isolates with $61 \%$ similarity.

\section{Physiological Characters}

\section{Osmophilia}

At $16.6 \%$ glycerol, all $S$. schenckii isolates were able to grow; at $20 \%$ glycerol, $89.47 \%$ of $S$. schenckii isolates grew (only one isolate from Central West and one isolate from the Southeast regions were inhibited); however, at $23 \%$ and $28.5 \%$, all isolates were inhibited (Table 3 ).

\section{Halophilia}

All isolates could grow at $6 \%$ and $8 \%$ salt $(\mathrm{NaCl})$ concentration. Eight (42.10\%) isolates (one from the North, three from the Northeast, one from the Central West, two from the Southeast, and one from the South region) could grow at $9 \%$ and $10 \%$ salt concentrations. No isolates grew at $12 \%$ (Table 3).

\section{pH Tolerance}

All isolates could grow between $\mathrm{pH} 2.2$ and 12.5, but none grew at $\mathrm{pH} 13.0$ (Table 3).

\section{Enzymatic Tests: Urease, DNAase, Proteinase, and Gelatinase Activities}

In relation to these enzymes, considered as virulence factors, all (100\%) S. schenckii isolates presented urease and DNAase activities. Only three (15.78\%) isolates (one from the North and two from the Southeast region) showed gelatinase activity, and five (26.31\%) isolates (one from the North, three from the Northeast, and one from the Southeast region) showed proteinase activities of $0.68,0.88,0.85,0.55$, and 0.78 , respectively. Also, only four $(21.05 \%)$ isolates (one from the North, one from the Central West, and two from the Southeast region) showed caseinase activities of $0.75,0.87,0.89$, and 0.87 , respectively (Table 3 ).

\section{DISCUSSION}

This study represents the first investigation of Brazilian S. schenckii isolates originating from different geographic regions of Brazil based on their phenotypic and genotypic characteristics. A high degree of similarity of the mean conidial area was observed among the isolates from the North, South, Southeast, Northeast, and Center West regions. There were no statistical differences in morphological structures related to geographical distribution or clinical forms of sporotrichosis among Brazilian S. schenckii 


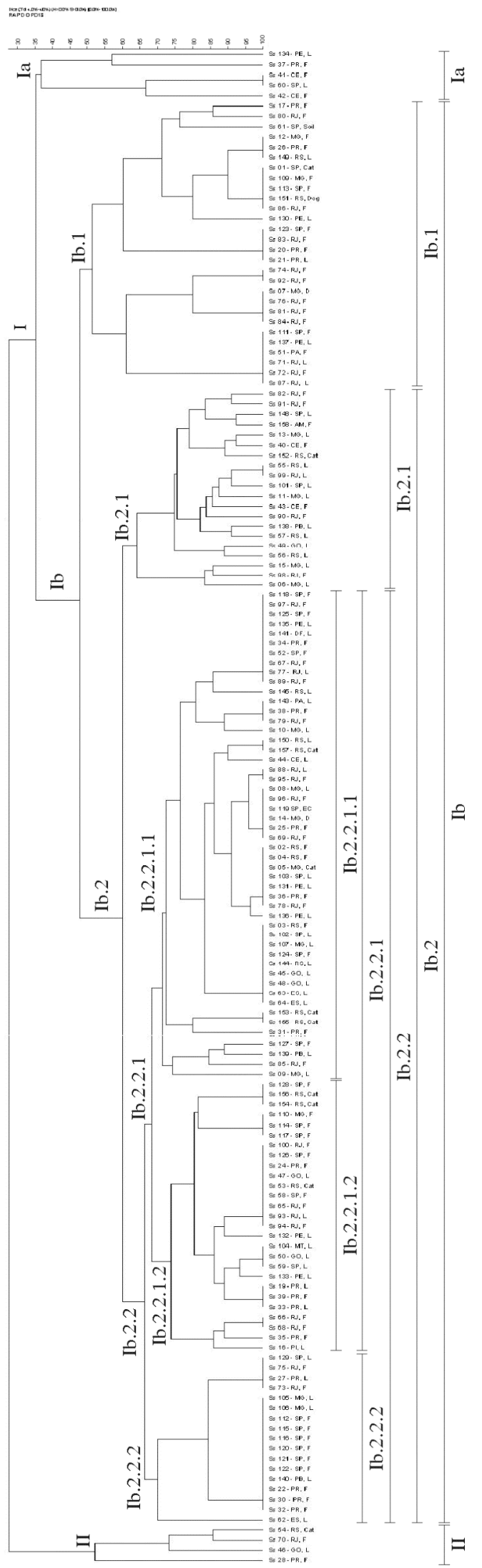

Fig. (1). Relationship of Brazilian S. schenckii isolates. The dendrogram was generated from genetic similarity coefficients obtained by determination of the presence or absence of DNA bands from 151 isolates and based on UPGMA.
Dice (Tol 2.0\% - 20\%) $(\mathrm{H}>0.0 \% \mathrm{~S}>0.0 \%)[0.0 \%-100.0 \%]$

RAPD OPD 18 - Säo Paulo state isolates

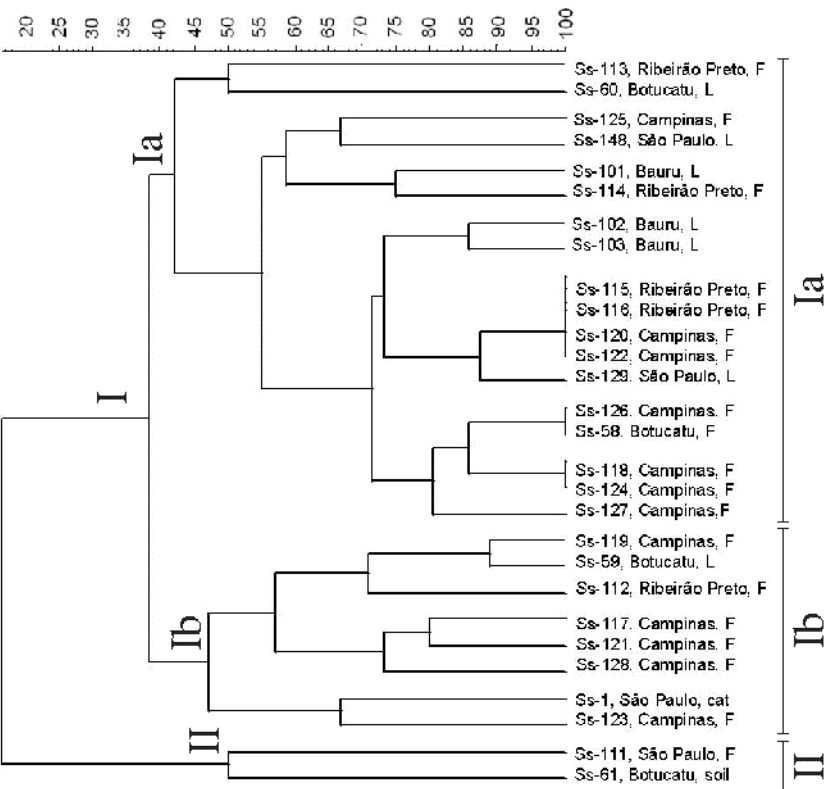

Fig. (2). Relationship among São Paulo State S. schenckii isolates. The dendrogram was generated from genetic similarity coefficients obtained by determination of the presence or absence of DNA bands from 28 isolates and based on UPGMA.

Dice (Tol $2.0 \%-2.0 \%)(\mathrm{H}>0.0 \% \mathrm{~S}>0.0 \%)[0.0 \%-100.0 \%]$

RAPD OPD 18 - Rio de Janeiro city isolates

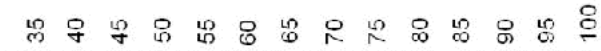

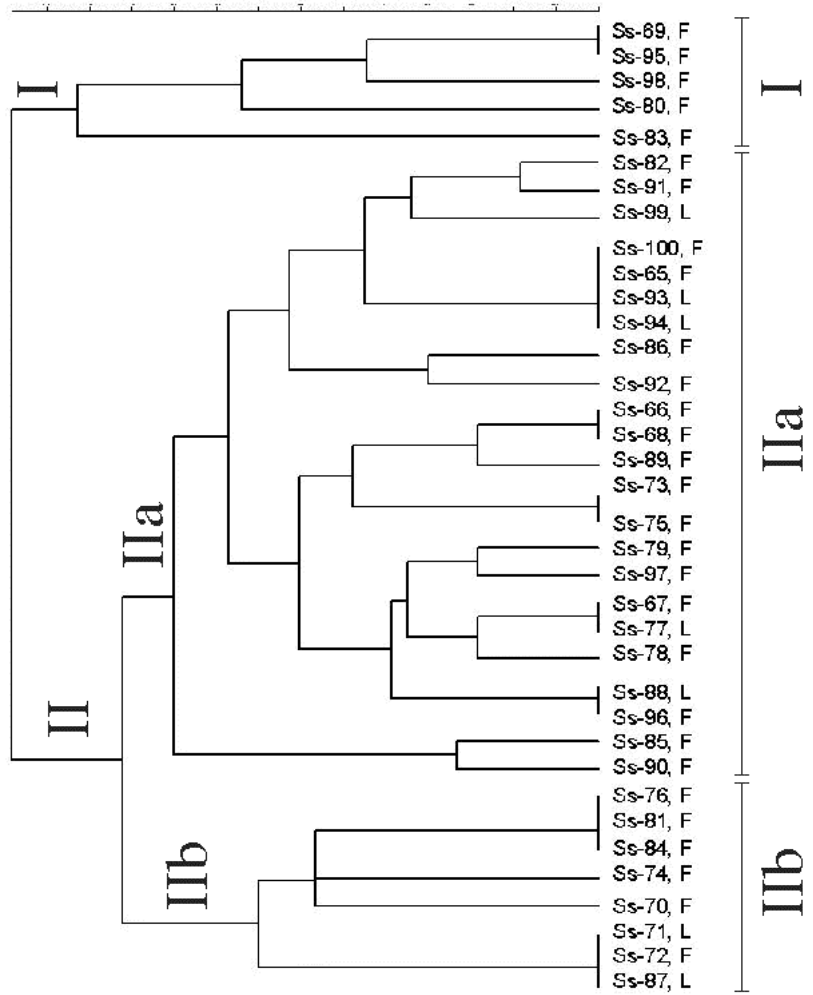

Fig. (3). Relationship among Rio de Janeiro city S. schenckii isolates. The dendrogram was generated from genetic similarity coefficients obtained by determination of the presence or absence of DNA bands from 36 isolates and is based on UPGMA. 
Table 3. Physiological Characters of $S$. schenckii Isolates

\begin{tabular}{|c|c|c|}
\hline Glycerol Concentration & $\%$ of Growth & $\%$ of Inhibition \\
\hline $16.6 \%$ & 100 & 0 \\
\hline $20.0 \%$ & 89.47 & 10.53 \\
\hline $23.0 \%$ & 0 & 100 \\
\hline $28.5 \%$ & 0 & 100 \\
\hline Salt Concentration & $\%$ of Growth & $\%$ of Inhibition \\
\hline $6 \%$ & 100 & 0 \\
\hline $8 \%$ & 100 & 0 \\
\hline $9 \%$ & 42.10 & 57.90 \\
\hline $10 \%$ & 42.10 & 57.90 \\
\hline $12 \%$ & 0 & 100 \\
\hline pH & $\%$ of Growth & $\%$ of Inhibition \\
\hline 2.2 & 100 & 0 \\
\hline 4.0 & 100 & 0 \\
\hline 12.0 & 100 & 0 \\
\hline 12.5 & 100 & 0 \\
\hline 13.0 & 0 & 100 \\
\hline Enzymes & \multicolumn{2}{|c|}{$\%$ of Activity Production } \\
\hline Urease & \multicolumn{2}{|c|}{100} \\
\hline DNAse & \multicolumn{2}{|c|}{100} \\
\hline Gelatinase & \multicolumn{2}{|c|}{15.78} \\
\hline Proteinase & \multicolumn{2}{|c|}{26.31} \\
\hline Caseinase & \multicolumn{2}{|c|}{21.05} \\
\hline
\end{tabular}

isolates. However, statistical differences were observed between animal and human S. schenckii isolates in terms of conidial area. Analyzing data for conidial sizes (width $\times$ length) described by Mesa-Arango et al. [22] and converting them to area $\left(\mu \mathrm{m}^{2}\right)$, we also verified that conidial area of $S$. schenckii from Mexico, Guatemala, and Colombia were greater than those obtained from Brazilian S. schenckii isolates (Mexico clinical isolates $=3.98 \mu^{2}$; Mexico environmental isolates $=4.54 \mu^{2}$; Guatemala clinical isolates = 7.69 $\mu^{2}$; Colombia clinical isolates $=7.20 \mu \mathrm{m}^{2}$; Brazilian isolates $=2.39 \mu \mathrm{m}^{2}$ ).

There are few reports on $S$. schenckii physiological characteristics in the literature $[23,26]$. In relation to osmophilia, seventeen isolates $(89.47 \%)$ could grow at glycerol concentrations $\leq 20 \%$. However, Ghosh et al. [23] showed that $100 \%$ of Indian isolates were able to grow at glycerol concentrations $\leq 20 \%$. In the halophilia assay, all Brazilian isolates grew very well at $6 \%$ and $8 \%$ salt, but only eight isolates $(42.10 \%)$ could grow at $9 \%$ and $10 \%$ salt concentrations, and none grew at $12 \%$. Sigler et al. [26] noted that four Canadian $S$. schenckii isolates were intolerant to a $7 \%$ salt concentration. However, all $S$. schenckii isolates studied by Ghosh et al. [23] in India were tolerant to $6 \%$ salt concentration although a portion could not grow at $7 \%$, and all were inhibited at $8 \%$ salt. Our data show that Brazilian $S$. schenckii isolates are more tolerant to high salt concentrations than are S. schenckii from other geographic regions of the world.

In relation to growth at different $\mathrm{pHs,} \mathrm{our} \mathrm{isolates} \mathrm{grew}$ very well between $\mathrm{pH} 2.2$ and $\mathrm{pH} 12.5$. Isolates from India grew well between $\mathrm{pH} 3.0$ and 11.5 , but only a few grew at $\mathrm{pH} 12.5$ [23]. On the other hand, in terms of enzymes considered as "virulence factors," all of our isolates produced urease and DNAase. Mendonza et al. [27] reported that all S. schenckii studied in Venezuela could produce urease. In addition, Ghosh et al. [23] in India reported that all mycelial forms of $S$. schenckii could split urea.

Gelatinase was produced only by three $(15.78 \%)$ of our isolates, one from the North region and two from the Southeast region, and caseinase was also produced by only four $(21.05 \%)$ isolates, one from the North region, two from the Southeast region and one from the Central West region. Ghosh et al. [23] showed that $49 \mathrm{~S}$. schenckii isolates from different regions of India were unable to hydrolyze gelatin or casein. Mendonza et al. [27] related that two out five $S$. schenckii isolates from Venezuela were positive for gelatinase activity. Proteinase was produced by five (26.31\%) of our isolates, one from the Southeast region, one from the North region, and three from the Northeast region. This kind of study is important for understanding the environmental and epidemiological aspects of S. schenckii by strain typing and possible selective isolation of this fungus [23].

Furthermore, the molecular studies with RAPD clearly identified no grouping of the Brazilian S. schenckii isolates based on geographical origins or the clinical form of sporotrichosis. The main clusters obtained did not show geographic separation, and different genotypes were present among them (isolates from distinct geographic regions may be present in a cluster). As observed in this study, the 67 PCR fingerprinting patterns generated showed a high discrimination among the $S$. schenckii strains. The high discrimination found among all Brazilian strains was also observed among the S. schenckii strains from Sao Paulo State and those from Rio de Janeiro, both of which are smaller geographical areas. The low similarity among Brazilian $S$. schenckii isolates indicate a high genetic variation in this species.

In keeping with our finding of no relationship between $S$. schenckii fingerprinting DNA profiles of our isolates with geographical area or clinical forms of the disease, MesaArango et al. [22] and Neyra et al. [28] also found no association of $S$. schenckii isolates with specific forms of sporotrichosis. Galhardo et al. [29] observed a great diversity in the PCR fingerprinting analysis results among $S$. schenckii isolates from Rio de Janeiro that they could not correlate with clinical forms. This finding suggested that the main factor determining clinical forms of sporotrichosis is more likely related to the immune condition of the patient and the route of transmission, rather than to the strain genotype [29]. Liu et al. [24] did correlate RAPD profiles among isolates recovered from patients with fixed cutaneous, lymphatic, and disseminated forms of sporotrichosis. However, in the present work, using the same primers as Liu et al. [24] and 
Mesa-Arango et al. [22], we did not correlate the DNA fingerprinting profiles with any clinical form or by geographic region.

The great diversity in DNA profiles found among the five distinct regions of Brazil was also confirmed within a smaller area of the country, Sao Paulo State, and the same level of diversity was confirmed in an even smaller area consisting of a single city, Rio de Janeiro. The similarities of these comparisons corroborate the idea that Brazilian $S$. schenckii is an organism with great genetic variability. These findings need to be further confirmed using more sensitive molecular approaches such as restriction fragment length polymorphism analysis with Southern blotting and sequencing of the calmodulin gene, which are already in progress in our laboratory.

\section{ACKNOWLEDGEMENTS}

We thank Fundação de Amparo à Pesquisa do Estado de São Paulo (FAPESP) and Conselho Nacional de Pesquisas (CNPq). We wish to thank the following people for generous provision of fungal isolates: Prof. Dr. Carlos Pelleschi Taborda (Sao Paulo University), Prof. Dr. Luis Carlos Severo (Federal University of Rio Grande do Sul), Prof. Dr. Júnia Soares Hadam (Federal University of Minas Gerais), Prof. Dr. Liline Maria Soares Martins (State University of Piaui), Prof. Dr. Flávio Queiroz-Telles (Federal University of Paraná), Prof. Dr. José Júlio Costa Sidrim (Federal University of Ceará), Prof. Dr. Marcos Fábio Gadelha Rocha (State University of Ceará), Prof. Dr. Maria do Rosário Rodrigues Silva (Federal University of Goiás), Prof. Dr. Patrícia Fagundes da Costa (Federal University of Pará), Prof. Dr. Arnaldo Lopes Colombo (Federal University of Sao Paulo), Prof. Dr. Mário Carlos Meireles Araújo (Federal University of Rio Grande), Prof. Dr. Eduardo Bagagli (UNESP, Botucatu), Prof. Dr. Mariceli Ribeiro (Federal University of Espirito Santo), Prof. Dr. Márcio Nucci (Federal University of Rio de Janeiro), Prof. Dr. Rosane Hahn (Federal University of Mato Grosso), Prof. Dr. Mário Léon Silva-Vergara (Faculty of Medicine of Triângulo Mineiro), Prof. Dr. Cláudia Maria Leite Maffei (Faculty of Medicine of Ribeirão Preto-FMRP-USP), Prof. Dr. Angélica Z. Schreiber (UNICAMP, Campinas), Prof. Dr. Cristina M. de Souza Motta (Federal University of Pernambuco), Prof. Dr. Edeltrudes de Oliveira Lima (Federal University of Paraíba), Prof. Dr. Silvia Helena Marques da Silva (Evandro Chagas Institute, Belém, Pará), Prof. Dr. Carmélia Matos Santiago Reis (Universidade de Brasília, UNB), and Prof. Dr. Maria Lúcia Scroferneck (Federal University of Rio Grande do Sul).

\section{REFERENCES}

[1] Kauffman CA. Sporothrichosis. Clin Infect Dis 1999; 29: 231-7.

[2] Kwon-Chung KJ, Bennett JE. Medical mycology. Philadelphia, PA: Lea \& Febiger 1992.

[3] Rippon JW. The pathogenic fungi and pathogenic actinomycetes. Medical Mycology. 3rd ed., Philadelphia: WB Saunders Company 1988; pp. 325-52.

[4] Mackinnon J, Conti-Diaz I, Gezuele E, Civila E, Luz S. Isolation of Sporothrix schenckii from nature and considerations on its pathogenicity and ecology. Sabouraudia 1969; 7: 38-45.
[5] Pappas PG, Telles I, Deep AE, Nolasco D, Holgado W, Bustamante B. Sporotrichosis in Peru: description of an area of hyperendemicity. Clin Infect Dis 2000; 30: 65-70.

[6] Barros MBL, Schubach A, Francesconi-do-Valle AC, et al. Cattransmitted sporotrichosis epidemic in Rio de Janeiro, Brazil: description of a series of cases. Clin Infect Dis 2004; 38: 529-35.

[7] Vismer HF, Hull PR. Prevalence, epidemiology and geographycal distribution of Sporothrix schenckii infections in Gauteng, South Africa. Mycopathologia 1977; 137: 137-43.

[8] González-Ochoa A. Contribuciones recientes al conocimiento de la esporotricosis. Gac Med Mex 1965; 95: 463-74.

[9] Muir DB, Pritchard RC. Sporothrix schenckii - incidence in the Sydney region. Aust J Dermatol 1984; 25: 27-8.

[10] Lyon GM, Zurita S, Casquero J, et al. Population-based surveillance and a case-control study of risk factors for endemic lymphocutaneous sporotrichosis in Peru. Clin Infect Dis 2003; 36: 34-9.

[11] Dixon DM, Salkin IF, Duncan RA, et al. Isolation and characterization of Sporothrix schenckii from clinical and environmental sources associated with the largest U.S. epidemic of sporotrichosis. J Clin Microbiol 1991; 29: 1106-13.

[12] Mariat F. Observation sur l'écologie de Sporothrix schenckii et Ceratocystes stenoceras en Corse and en Alsace, provinces françaises indemnes de sporotrichose. Sabouraudia 1975; 13: 21725.

[13] Donadel K, Reinoso Y, Oliveira J, Azulai RD. Esporotricose: revisão. An Brasil Dermatol 1993; 68: 45-52.

[14] Fleury RN, Taborda PR, Gupta AK, et al. Zoonotic sporotrichosis. Transmission to humans by infected domestic cat scratching: report of four cases in São Paulo, Brazil. Int J Dermatol 2001; 40: 318-22.

[15] Marques SA, Franco SR, Camargo RM, Dias LD, Haddad Junior V, Fabris VE. Sporotrichosis of the domestic cat (Felis catus): human transmission. Rev Inst Med Trop São Paulo 1993; 35: 32730.

[16] Rosa ACM, Scroferneker ML, Vettorato R, Gervini RL, Vettorato G, Weber A. Epidemiology of sporotrichosis: a study of 304 cases in Brazil. J Am Acad Dermatol 2005; 52: 451-9.

[17] Schubach TM, Schubach A, Okamoto T, et al. Evaluation of an epidemic of sporotrichosis in cats: 347 cases (1998-2001). J Am Vet Med Assoc 2004; 224: 1623-9.

[18] Schubach TMP, Schubach A, Okamoto T, et al. Canine sporotrichosis in Rio de Janeiro, Brazil: clinical presentation, laboratory diagnosis and therapeutic response in 44 cases (19982003). Med Mycol 2006; 44: 87-92.

[19] Marimon R, Cano J, Gené J, Sutton DA, Kawasaki M, Guarro J. Sporothrix schenckii, S. globosa, and S. mexicana, three new Sporothrix species of clinical interest. J Clin Microbiol 2007; 45: 3198-206.

[20] Marimon R, Gené J, Cano J, Trilles L, Lazéra MS, Guarro J. Molecular phylogeny of Sporothrix schenckii. J. Clin Microbiol 2006; 44: 3251-6.

[21] Meyer EM, Beer ZW, Summerbell RC, et al. Taxonomy and phylogeny of new wood- and soil- inhabiting Sporothrix species in the Ophiostoma stenoceras-Sporothrix complex. Mycologia 2008; 100: 647-61.

[22] Mesa-Arango AC, Reyes-Montes MR, Pérez-Mejía A, et al. Phenotyping and genotyping of Sporothrix schenckii isolates according to geographical origin and clinical form of sporotrichosis. J Clin Microbiol 2002; 40: 3004-11.

[23] Ghosh A, Maity PK, Hemashettar BM, Sharma VK, Chakrabarti A. Physiological characters of Sporothrix schenckii isolates. Mycoses 2002; 45: 449-54.

[24] Liu X, Lian C, Jin L, An L, Yang G, Lin X. Characterization of Sporothrix schenckii by random amplification of polymorphic DNA assay. Chin Med J 2003; 116: 239-42.

[25] Price MF, Wilkinson ID, Gentry LO. Plate method for detection of phospholipase activity in Candida albicans. Sabouraudia 1982; 20: 7-14.

[26] Sigler L, Harris JL, Dixon DM, et al. Microbiology and potential virulence of $S$. schenckii, a fungus rarely isolated from blood and skin. J Clin Microbiol 1990; 28: 1009-115. 
[27] Mendonza M, Alvarado P, Torrez ED, Lucena L, Albornoz MC. Comportamiento físiológico y de sensibilidad in vitro de aislamientos de Sporothrix schenckii mantenidos 18 años por dos métodos de presevación. Rev Iberoam Micol 2005; 22: 151-6.

[28] Neyra E, Fonteyne P-A, Swinne D, Fauche F, Bustamante B, Nolard N. Epidemiology of human sporotrichosis investigated by amplified fragment length polymorphism. J Clin Microbiol 2005; 43: $1348-52$

[29] Galhardo MC, Oliveira RMZ, Valle ACF, et al. Molecular epidemiology and antifungal susceptibility patterns of Sporothrix schenckii isolates from a cat-transmitted epidemic of sporotrichosis in Rio de Janeiro, Brazil. Med Mycol 2008; 46: 141-51.

(C) Fernandes et al.; Licensee Bentham Open.

This is an open access article licensed under the terms of the Creative Commons Attribution Non-Commercial License (http://creativecommons.org/licenses/by$\mathrm{nc} / 3.0 /$ ) which permits unrestricted, non-commercial use, distribution and reproduction in any medium, provided the work is properly cited. 